\title{
Análisis de la evolución de los operados mediante cirugía de mínimo acceso en nuestro hospital. ¿Presenta mejores resultados que la convencional?
}

\author{
Analysis of the evolution of those operated on with minimal access surgery in our \\ hospital. Does it present better results than the conventional one? \\ Nora García*, Aníbal Bermúdez, Ma. Ángeles Martín, Carmen Carmona, Cristina Jaén y Tomás Daroca \\ Servicio Cirugía Cardiovascular, Hospital Puerta del Mar, Cádiz, España
}

\begin{abstract}
Resumen
La estenosis aórtica severa sintomática es la patología quirúrgica más prevalente en cirugía cardiaca y su sustitución aislada se ha realizado históricamente mediante esternotomía media completa. Sin embargo, se ha producido recientemente una gran revolución, especialmente tras la llegada de las prótesis aórticas sin suturas que, unido a un nuevo impulso por la cirugía cardiaca hacia un rumbo menos invasivo, ha provocado que el reemplazo de dicha válvula se lleve a cabo cada vez más frecuentemente por dichas prótesis y por incisiones de mínimo acceso. Por ello, realizamos una revisión de los casos intervenidos en nuestro servicio desde el inicio del programa de cirugía de mínimo acceso comparándolos con los resultados de los casos intervenidos en la misma época mediante cirugía convencional.
\end{abstract}

Palabras claves: Cirugía. Mínimo acceso. Convencional. Comparación. Resultados.

\begin{abstract}
Symptomatic severe aortic stenosis is the most prevalent surgical pathology in cardiac surgery, and its isolated replacement has historically been performed by means of complete middle sternotomy. However, a great revolution has recently taken place, especially after the arrival of sutureless aortic prostheses that, together with a new impulse by cardiac surgery towards a less invasive course, has caused the replacement of said valve to be carried out more and more frequently due to these prostheses and minor access incisions. For this reason, we carried out a review of the cases operated on in our service from the beginning of the minimum access surgery program, comparing them with the results of the cases operated at the same time using conventional surgery.
\end{abstract}

Key words: Surgery. Minimal access. Conventional. Comparison. Results. 


\section{Introducción}

La estenosis aórtica representa la patología valvular con mayor indicación quirúrgica y se realiza históricamente mediante esternotomía convencional. Sin embargo, desde los comienzos de la cirugía cardiaca surgió el interés por emplear vías menos invasivas, aunque no experimentó una gran aceptación. No obstante, en los últimos años la cirugía cardiaca ha experimentado el resurgimiento y globalización de la cirugía de mínimo acceso, debido $a^{1}$ :

- El crecimiento de la hemodinámica intervencionista con el tratamiento percutáneo de varias patologías, sobre todo en la válvula aórtica, que ha provocado que la cirugía cardiaca deba actualizarse para poder competir con ella.

- La estandarización de los pasos en la cirugía mínimamente invasiva con un abordaje mayoritario en la válvula aórtica que es la miniesternotomía en $\mathrm{J}$, con la publicación de los resultados experimentados en cada centro, demostrando que es una alternativa real ${ }^{1}$.

- La llegada de las prótesis aórticas sin suturas, que permiten acortar el tiempo necesario en la implantación de dicha prótesis y el tiempo total de isquemia cardiaca.

- El envejecimiento y el aumento de la comorbilidad de los pacientes que se derivan a cirugía, por lo que se intenta ser lo menos agresivo posible quirúrgicamente.

Por otra parte, en la literatura, la cirugía de mínimo acceso presenta numerosas ventajas en la evolución del paciente, como: menor necesidad de ventilación mecánica y menor aparición de complicaciones respiratorias, así como una estancia más corta en la unidad de cuidados críticos ( $\mathrm{UCl}$ ) y menor duración del ingreso hospitalario. Por todo ello, con la intención de poner en marcha un programa de cirugía de mínimo acceso, se comenzó a llevar a cabo la sustitución valvular aórtica aislada mediante una miniesternotomía en J por el $3^{\text {er- }}$ $4^{\circ}$ espacio intercostal, ya que resultaba la incisión más utilizada según la literatura y porque es el abordaje que más se asemeja a la esternotomía completa, además de que permite llevar a cabo más fácilmente la reconversión a técnica convencional, entre otras ventajas. Con el objetivo de analizar los resultados de esta técnica en nuestro medio y descubrir si se equiparan o no a los resultados en otros centros, se ha realizado este estudio retrospectivo que compara la evolución de los pacientes sometidos a reemplazo valvular aórtico dividiéndolos en dos grandes grupos: el grupo de la miniesternotomía y el de la esternotomía completa ${ }^{1,2}$.

\section{Métodos}

Se llevó a cabo un estudio observacional analítico retrospectivo de evaluación de una intervención no deliberada, ya que para su análisis se ha dividido a los pacientes en dos grupos: el grupo 1, que se refiere a la sustitución valvular aórtica mediante prótesis Perceval ${ }^{\circledR}$ por miniesternotomía en J, y el grupo 2, que es la sustitución valvular por prótesis biológicas soportadas mediante esternotomía convencional, localizado en el Servicio de Cirugía Cardiovascular del Hospital Puerta del Mar entre enero de 2016 y diciembre de 2019, con un total de 57 pacientes en cada grupo.

En cuanto al análisis estadístico, todos los datos obtenidos introdujeron y procesaron en una base de datos anonimizada y encriptada en formato Exce $^{\circledR}$. Se exportaron a un archivo de datos Statgraphics ${ }^{\circledR}$ y posteriormente se analizaron los datos obtenidos mediante programas y aplicaciones de tipo estadístico (SPSS ${ }^{\circledR}$ y $\mathrm{R})$, realizando el análisis de la varianza para averiguar si existían diferencias estadísticamente significativas entre los dos grupos. Las variables se muestran en la tabla 1.

\section{Resultados}

En cuanto a las variables sociodemográficas, la variable sexo resultó estadísticamente significativa, con mayor porcentaje de la categoría mujeres en el grupo de miniesternotomía. Con respecto a las variables de comorbilidades, se obtuvieron una mayor proporción de sujetos hipertensos, dislipidémicos, con enfermedad pulmonar crónica obstructiva (EPOC) y diabéticos en el grupo de miniesternotomía. La única proporción que resultó superior en el grupo 2 fue la de fumadores, sin ser ninguna de las anteriores estadísticamente significativas.

Por otro lado, en las variables complementarias, la estancia en $\mathrm{UCl}$ y la estancia hospitalaria fueron mayores en el grupo de miniesternotomía, con una media de 5.65 días frente $3.58 \mathrm{U:} 1,139 ; p=0.004)$, y una media de 15.67 días frente a 13.04 (U: 1,201.5; $p=0.016)$, es decir, estadísticamente significativas. Además, se obtuvieron mayor proporción de pacientes reintervenidos por sangrado en las primeras 24 horas, reintervenidos por otras causas y desarrollo de mediastinitis en el grupo de miniesternotomía frente al de esternotomía, y tras el cálculo con $\chi^{2}$, de estas últimas solo resultaron estadísticamente significativas la reintervención por sangrado y la reintervención por otras 
Tabla 1. Variables del estudio

\begin{tabular}{|c|c|c|c|}
\hline Variables sociodemográficas & Tipo de variable & naturaleza estadística & Categoría \\
\hline Edad & Independiente & Cuantitativa medida en años & \\
\hline Sexo & Independiente & Cualitativa dicotómica & $\begin{array}{l}\text { - Hombre } \\
\text { - Mujer }\end{array}$ \\
\hline $\begin{array}{l}\text { Variables de comorbilidades } \\
\text { Tabaquismo } \\
\text { Superficie corporal } \\
\text { Hipertensión arterial } \\
\text { Diabetes mellitus } \\
\text { Dislipemia } \\
\text { EPOC }\end{array}$ & $\begin{array}{l}\text { Independiente } \\
\text { Independiente } \\
\text { Independiente } \\
\text { Independiente } \\
\text { Independiente } \\
\text { Independiente }\end{array}$ & $\begin{array}{l}\text { Cualitativa dicotómica } \\
\text { Cuantitativa medida en } \mathrm{m}^{2} \\
\text { Cualitativa dicotómica } \\
\text { Cualitativa dicotómica } \\
\text { Cualitativa dicotómica } \\
\text { Cualitativa dicotómica }\end{array}$ & $\begin{array}{l}\text { - Sí } \\
\text { - No } \\
\text { - Sí } \\
\text { - No } \\
\text { - Sí } \\
\text { - No } \\
\text { - Sí } \\
\text { - No } \\
\text { - Sí } \\
\text { - No }\end{array}$ \\
\hline $\begin{array}{l}\text { Tiempo de isquemia cardiaca } \\
\text { Estancia en la unidad de cuidados críticos } \\
\text { Estancia total hospitalaria } \\
\text { Mortalidad postoperatoria ( } 30 \text { días) } \\
\text { Ritmo cardiaco } \\
\text { Mediastinitis } \\
\text { Sangrado en las primeras } 24 \text { h } \\
\text { Necesidad de reintervención urgente por sangrado } \\
\text { Necesidad de reintervención urgente por otras } \\
\text { causas } \\
\text { Tipo de lesión valvular }\end{array}$ & $\begin{array}{l}\text { Independiente } \\
\text { Independiente } \\
\text { Independiente } \\
\text { Independiente } \\
\text { Independiente } \\
\text { Independiente } \\
\text { Independiente } \\
\text { Independiente } \\
\text { Independiente } \\
\text { Independiente }\end{array}$ & $\begin{array}{l}\text { Cuantitativa medida en minutos } \\
\text { Cuantitativa medida en días } \\
\text { Cuantitativa medida en días } \\
\text { Cualitativa dicotómica } \\
\text { Cualitativa policotómica } \\
\text { Cualitativa dicotómica } \\
\text { Cuantitativa medida en mililitros } \\
\text { Cualitativa dicotómica } \\
\text { Cualitativa dicotómica } \\
\text { Cualitativa policotómica }\end{array}$ & $\begin{array}{l}\text { - Sí } \\
\text { - No } \\
\text { - Sinusal } \\
\text { - Fibrilación auricular } \\
\text { - Marcapasos } \\
\text { - Sí } \\
\text { - No } \\
\text { - Sí } \\
\text { - No } \\
\text { - Sí } \\
\text { - No } \\
\text { - Estenosis } \\
\text { - Regurgitación } \\
\text { - Ambas }\end{array}$ \\
\hline $\begin{array}{l}\text { Variables del estudio } \\
\text { Tiempo de intubación } \\
\text { Complicaciones respiratorias }\end{array}$ & $\begin{array}{l}\text { Dependiente } \\
\text { Dependiente }\end{array}$ & $\begin{array}{l}\text { Cualitativa dicotómica } \\
\text { Cualitativa policotómica }\end{array}$ & $\begin{array}{l}\text { - Más de } 24 \text { horas } \\
\text { - Menos de } 24 \text { horas } \\
\text { - Sin complicaciones } \\
\text { - Derrame pleural } \\
\text { - Atelectasia } \\
\text { - Neumonía } \\
\text { - Insuficiencia respiratoria } \\
\text { - Distrés respiratorio }\end{array}$ \\
\hline
\end{tabular}

EPOC: enfermedad pulmonar obstructiva crónica; NYHA: New York Heart Association.

causas ( $p=0.052$ y $p=0.018$, respectivamente), como se muestra en la tabla 2.

Por último, con respecto a las variables principales del estudio, las complicaciones respiratorias y el tiempo de intubación, se obtuvo que fue superior la proporción de pacientes del grupo de esternotomía que no presentó ninguna complicación respiratoria, un 80.7 frente al $68.9 \%$ del grupo de miniesternotomía. También fue superior la proporción de pacientes del grupo de esternotomía que permanecieron intubados menos de 24 horas. No obstante, al aplicar la $\chi^{2}$ con respecto a cada grupo, no se obtuvieron diferencias 
Tabla 2. Análisis bivariante

\begin{tabular}{|c|c|c|c|}
\hline Variables complementarias & Miniesternotomía ( $\mathrm{n}=57$ ) & Esternotomía ( $\mathrm{n}=57$ ) & $p(p<0.05)$ \\
\hline Tiempo de clampaje aórtico & $60.44(56.07-65.80)$ & 64 (58.42-69.58) & 0.293 \\
\hline Sangrado por drenajes $24 \mathrm{~h}$ & $247.02(202.27-291.76)$ & $285.79(235.23-336.35)$ & 0.357 \\
\hline Estancia unidad de cuidados críticos & $5.65(4.16-7.14)$ & $3.58(2.52-4.64)$ & 0.004 \\
\hline Estancia total hospitalaria & $15.67(13.27-18.07)$ & $13.04(11.20-14.87)$ & 0.016 \\
\hline Mortalidad & $1(1.8 \%)$ & $3(5.3 \%)$ & 0.309 \\
\hline Reintervención por sangrado & $11(19.3 \%)$ & $4(7 \%)$ & 0.052 \\
\hline Reintervención por otras causas & $13(22.8 \%)$ & $4(7 \%)$ & 0.018 \\
\hline Medistinitis & $2(3.5 \%)$ & $0(0 \%)$ & 0.154 \\
\hline $\begin{array}{c}\text { NYHA } \\
\text { I } \\
\text { II } \\
\text { III } \\
\text { IV }\end{array}$ & $\begin{array}{c}0(0 \%) \\
32(56.1 \%) \\
25(43.9 \%) \\
0(0 \%)\end{array}$ & $\begin{aligned} & 1(1.8 \%) \\
33 & (57.9 \%) \\
22 & (38.6 \%) \\
1 & (1.8 \%)\end{aligned}$ & 0.531 \\
\hline $\begin{array}{l}\text { Fracción de eyección del ventrículo izquierdo } \\
\text { Conservada } \\
\text { Disminuida }\end{array}$ & $\begin{array}{c}51(89.5 \%) \\
6(10.5 \%)\end{array}$ & $\begin{array}{c}57(100 \%) \\
0(0 \%)\end{array}$ & 0.012 \\
\hline $\begin{array}{l}\text { Ritmo cardiaco } \\
\text { Sinusal } \\
\text { Fa } \\
\text { Marcapasos }\end{array}$ & $\begin{array}{c}52(91.2 \%) \\
4(7 \%) \\
1(1.8 \%)\end{array}$ & $\begin{array}{l}47(82.5 \%) \\
7(12.3 \%) \\
3(5.3 \%)\end{array}$ & 0.355 \\
\hline $\begin{array}{l}\text { Tipo lesión valvular } \\
\text { Estenosis } \\
\text { Regurgitación } \\
\text { Mixta }\end{array}$ & $\begin{array}{c}45(78.9 \%) \\
2(3.5 \%) \\
10(17.5 \%)\end{array}$ & $\begin{array}{c}43(75.4 \%) \\
1(1.8 \%) \\
13(22.8 \%)\end{array}$ & 0.680 \\
\hline Variables del estudio & Miniesternotomía ( $\mathrm{n}=57$ ) & Esternotomía ( $=57$ ) & $p(p<0.05)$ \\
\hline $\begin{array}{l}\text { Tiempo de intubación } \\
<24 \mathrm{~h} \\
>24 \mathrm{~h}\end{array}$ & $\begin{array}{c}48(84.2 \%) \\
9(15.8 \%)\end{array}$ & $\begin{array}{c}51(89.5 \%) \\
6(10.5 \%)\end{array}$ & 0.406 \\
\hline $\begin{array}{l}\text { Complicaciones respiratorias } \\
\text { Sin complicaciones } \\
\text { Derrame pleural } \\
\text { Neumonía } \\
\text { Insuf. resp. } \\
\text { Atelectasia } \\
\text { Distrés } \\
\text { Neumonía }\end{array}$ & $\begin{array}{c}39(68.9 \%) \\
8(14 \%) \\
5(8.8 \%) \\
0(0 \%) \\
4(7 \%) \\
1(1.8 \%) \\
5(8.8 \%)\end{array}$ & $\begin{array}{c}46(80.7 \%) \\
7(12.3 \%) \\
1(1.8 \%) \\
1(1.8 \%) \\
2(3.5 \%) \\
0(0 \%) \\
1(1.8 \%)\end{array}$ & $\begin{array}{l}0.309 \\
\text { Neumonía } \\
\text { Insuf. resp. } \\
\text { Atelectasia } \\
\text { Distrés } \\
\text { Neumonía }\end{array}$ \\
\hline
\end{tabular}

NYHA: New York Heart Association.

estadísticamente significativas. Además, la variable mortalidad fue superior, aunque ligeramente y sin significación, en el grupo de esternotomía, concretamente un 5.3 frente a un $1.8 \%$, lo que refleja que a pesar de presentar mayor ingreso en $\mathrm{UCl}$ y en el hospital y mayor incidencia de eventos respiratorios, el grupo de miniesternotomía evolucionó de forma más favorable que el grupo de esternotomía, como se refleja en la tabla 2.

\section{Discusión}

La medicina en general y la cirugía en particular han evolucionado mucho en los últimos años con la incorporación de procedimientos diagnósticos y terapéuticos menos invasivos, como por ejemplo con la cirugía laparoscópica utilizada de forma sistemática en la extracción de vesículas y/o en las intervenciones sobre el colon, debido a dos razones fundamentales: 
- Llevar a cabo los procedimientos con menor agresión y menor incisión quirúrgica implica menor dolor postoperatorio, menor ingreso hospitalario (con menor coste de este) y un retorno más rápido a la vida habitual del paciente, incluido el ámbito laboral ${ }^{2}$.

- El envejecimiento de la población con aumento de la comorbilidad de esta provoca el intento de ser lo menos invasivo posible para disminuir el riesgo quirúrgico y del postoperatorio.

- No hay que olvidar una tercera razón, no menos importante, que es el deseo del paciente por una incisión más estética.

Por todo ello, y por la constante innovación en otras ramas quirúrgicas, la cirugía cardiaca desde su inicio también intentó desarrollar técnicas mínimamente invasivas. Sin embargo, no obtuvieron el éxito esperado y la generalización de esta nueva corriente fue prácticamente nula. Actualmente, toda esta situación ha dado un giro y desde hace unos pocos años se ha producido una gran revolución que ha impulsado de nuevo a la cirugía cardiaca de mínimo acceso, gracias sobre todo a la estandarización y generalización de los pasos quirúrgicos, mostrando que es una técnica con una curva de aprendizaje necesaria pero rápidamente asumible. Con el objetivo de analizar cuáles son los resultados con el abordaje mínimamente invasivo en nuestro medio, concretamente en nuestro hospital, donde empezó a realizarse de forma sistemática a partir de 2016, se ideó este estudio, centrándonos en dos ítems: el tiempo de intubación de los pacientes con cada técnica y las complicaciones respiratorias asociadas a cada abordaje. Ambos ítems resultan muy relevantes en el postoperatorio de cirugía cardiaca, ya que marcan la estancia de los pacientes en $\mathrm{UCl}$, la aparición de complicaciones respiratorias y de otros tipos, la necesidad de reingreso en $\mathrm{UCl} y$, en general, la morbilidad y mortalidad en el postoperatorio. En la literatura consultada se han encontrado los siguientes resultados en cuanto a dichos ítems:

- En el estudio de Filip, et al. la media de tiempo de intubación en el grupo de miniesternotomía fue de 8 horas, mientras que la media del grupo de esternotomía fue de 10 horas, con una diferencia estadísticamente significativa $(p=0.045)$ a favor de la miniesternotomía. Sin embargo, en este estudio no se encontraron diferencias en cuanto a las complicaciones respiratorias entre ambos grupos.

- En el estudio de Young, et al. se obtuvo una diferencia de 2 horas en la media del tiempo de intubación entre ambas técnicas, a favor de la miniesternotomía.
- En el metaanálisis de Phan, et al. se observó una reducción del tiempo de intubación de media de 4 horas a favor del grupo de miniesternotomía.

Estos resultados no fueron así en nuestro estudio, ya que no se encontraron diferencias estadísticamente significativas con respecto al tiempo de intubación y las complicaciones respiratorias entre ambos grupos como era de esperar por los estudios consultados, es más, el grupo de miniesternotomía experimentó mayor número de eventos respiratorios. No se puede atribuir a que los grupos eran diferentes y que comprendían pacientes más graves, ya que no se obtuvieron diferencias estadísticamente significativas en el análisis de las variables de comorbilidades.

En cuanto al sangrado por los drenajes en las primeras 24 horas del postoperatorio, que se presupone que es inferior en las técnicas mínimamente invasivas al provocar menor agresión, ha resultado una variable difícil de valorar en la literatura, puesto que en la mayoría de los estudios no especifican esta cantidad, sino que hablan de la necesidad de transfusión, pero sí se ha encontrado lo siguiente:

- En el estudio de Filip, et al. el débito por los drenajes fue de una media de $230 \mathrm{ml}$ menor en el grupo de miniesternotomía con respecto al grupo de esternotomía. No obstante, no especifican si utilizaron medicación procoagulante.

En nuestro estudio, la media del sangrado fue menor en el grupo de miniesternotomía $(285,79 \mathrm{ml})$, pero no resultó estadísticamente significativa.

Uno de los puntos fuertes a favores de la miniesternotomía, al menos teóricamente, es que se relaciona con una menor estancia en $\mathrm{UCl}$ y menor estancia total hospitalaria, como se observa en el estudio de Phan, et al., con una media de reducción de ingreso en $\mathrm{UCl}$ de 0.6 días para el grupo miniesternotomía. Sin embargo, en nuestro estudio no se pudo demostrar esta diferencia, es más, resultó a favor del grupo esternotomía, con una estancia media en UCI de 3.58 días frente a 5.65 días en el grupo de miniesternotomía $(p=0.004)$, mientras que el grupo de esternotomía presentaba una estancia media en el hospital de 13,04 frente a 15,67 días del grupo de miniesternotomía ( $p=0.016)$. Es probable que estos resultados en nuestro estudio se deban a la curva de aprendizaje en la técnica mínimamente invasiva, tanto en el quirófano como en su manejo en $\mathrm{UCl}$, y a que los primeros casos presentaron mayor incidencia de complicaciones, que alargaron el ingreso de dichos pacientes.

Por otra parte, la variable mortalidad fue superior, aunque ligeramente y sin significación estadística, en 
el grupo de esternotomía con respecto al de miniesternotomía, concretamente un 5.3 frente a un $1.8 \%$, lo que refleja que a pesar de presentar mayor ingreso en $\mathrm{UCl}$ y en el hospital y mayor incidencia de eventos respiratorios, el grupo de miniesternotomía evolucionó de forma más favorable que el grupo de esternotomía. En el estudio de Paredes, et al. la mortalidad del grupo de miniesternotomía fue prácticamente del $0 \%$, mientras que la del grupo de esternotomía fue del $5 \%{ }^{3}$.

Por tanto, en nuestro análisis no se ha podido demostrar la presencia de las ventajas de la cirugía mínimamente invasiva descritas por otros estudios, especialmente en cuanto a la mortalidad (como en el de Paredes, et al.), ni en la estancia en UCl y de ingreso total (como en el estudio de Phan, et al.). Tampoco se pudo obtener una mayor muestra, como en el estudio de Cánovas, et al., en el que reclutaron a 963 pacientes sometidos a sustitución valvular aórtica por miniesternotomía en su mayor parte, gracias a la colaboración de varios centros, aunque los resultados de dicho estudio se centraron en las técnicas de mínimo acceso y no las compararon con los resultados de la técnica convencional, por lo que se pudo determinar que la cirugía cardiaca de mínimo acceso es una técnica segura, pero no si es mejor, peor o igual que la cirugía convencional. Por ello, en nuestro análisis el objetivo principal era comparar la evolución de los pacientes con ambas técnicas ${ }^{1,3}$.

\section{Conclusión}

En conclusión, en nuestro análisis no se ha podido demostrar que la cirugía de mínimo acceso presenta mejores resultados con respecto a la técnica convencional, probablemente por la naturaleza retrospectiva del estudio, el tamaño de la muestra y, sobre todo, la curva de aprendizaje asociada a la implementación de una nueva técnica. Lo más probable es que, actualmente, se si ideara un estudio prospectivo en nuestro servicio comparando ambas técnicas, tras pasar la curva de aprendizaje, obtuviésemos otros resultados bien distintos que demostrasen que la cirugía de mínimo acceso es una alternativa real a la cirugía convencional y que, en un futuro muy cercano, será la técnica estándar no solo en la válvula aórtica.

\section{Financiamiento}

La presente investigación no ha recibido ninguna beca específica de agencias de los sectores público, comercial, o sin ánimo de lucro.

\section{Conflicto de intereses}

\section{Ninguno.}

\section{Responsabilidades éticas}

Protección de personas y animales. Los autores declaran que para esta investigación no se han realizado experimentos en seres humanos ni en animales.

Confidencialidad de los datos. Los autores declaran que en este artículo no aparecen datos de pacientes.

Derecho a la privacidad y consentimiento informado. Los autores declaran que en este artículo no aparecen datos de pacientes.

\section{Bibliografía}

1. Paredes FA, Cánovas SJ, Gil Ó, García-Fuster R, Hornero F, Vázquez A et al. Cirugía mínimamente invasiva para el recambio valvular aórtico. Una técnica segura y útil más allá de lo estético. Rev Española Cardiol. 2013;66(9):695-9

2. Khoshbin E, Prayaga S, Kinsella J, Sutherland FW. Mini-sternotomy for aortic valve replacement reduces the length of stay in the cardiac intensive care unit: meta-analysis of randomised controlled trials. BMJ Open. 2011;1(2):e000266.

3. Rojas SV, Haverich A. Cirugía cardiaca mínimamente invasiva: ¿una alternativa segura para pacientes que requieren recambio valvular aórtico? Rev Esp Cardiol. 2013;66(9):685-6. 